\title{
Antimicrobial Resistance in ESBL-Producing Escherichia coli Isolated from Layer and Pig Farms in Thailand
}

\author{
Aniroot Nuangmek ${ }^{1,2}$, Suvichai Rojanasthien ${ }^{3}$, Suwit Chotinun ${ }^{3}$, Panuwat Yamsakul ${ }^{3}$, \\ Pakpoom Tadee ${ }^{3}$, Visanu Thamlikitkul ${ }^{4}$, Nattasit Tansakul ${ }^{5}$ \& Prapas Patchanee ${ }^{3}$
}

\begin{abstract}
Background: Study of drug resistance of commensal bacteria in both humans and animals can determine the scale of the drug resistance problem. Usage of antimicrobials to treat infections in humans and animals has generated extensive antimicrobial pressure not only on targeted pathogens but also on commensal bacteria. Commensal Escherichia coli appears to be the major reservoir for resistant genes implicated in the transmission of genetic traits from one bacterium to another. Antimicrobial resistance in Enterobacteriaceae has increased dramatically worldwide in the last decade. An increasing number of community-onset extended-spectrum beta-lactamase (ESBL)-producing bacterial infections, especially those caused by ESBL-producing E. coli, have been reported in many countries, including Thailand. Moreover, ESBL-producing E. coli have been widely detected in food-producing animals and the environment. The increased use of ESBLs in food animals is a serious public health problem. The objective of the study was to determine the prevalence and antimicrobial resistance pattern of ESBL-producing E. coli isolated from pigs, layers, farm workers and stagnant water, in order to increase awareness about antimicrobial usage on farms and to minimize the expansion of the antimicrobial resistance phenomenon in farm settings.

Materials, Methods \& Results: A total of 588 samples were collected from 107 pig farms and 89 layer farms in Chiang Mai-Lamphun and Chon Buri provinces during May 2015-April 2016. Double-disk diffusion method according to EUCAST (European Committee on Antimicrobial Susceptibility Testing) guidelines was used for detection. The results demonstrated that $36.7 \%$ (216/588) of samples were ESBL-producing E. coli-positive, including rectal swabs $74.8 \%$ (80/107), pig farm worker stool swabs $57.0 \%$ (61/107), stagnant water on pig farms $21.5 \%$ (23/107), healthy layer rectal swabs 6.7\% (6/89) and layer farm worker stool swabs 51.7\% (46/89). Most of the isolates were resistant against ampicillin (99.5\%), followed by erythromycin $(98.6 \%)$ and ceftriaxone $(96.3 \%)$. All of them were classified as multidrug-resistant strains. Moreover, AMP-CRO-E-TE-C-SXT-CN was the most frequent phenotype pattern detected in animals, humans and the environment, followed by AMP-CRO-E-TE-C-SXT-NA-CN.

Discussion: The present study offers clear evidence that the prevalence of ESBL-producing E. coli in healthy pigs is higher than in layers. One possible explanation is that a large amount and variety of antimicrobials are used on pig farms, resulting in a common and significant source of drug-resistant ESBL-producing E. coli. The lower incidence of ESBL-producing E. coli in samples from a pig farm environment than in samples of animal origin indicate that pigs are a reservoir of a reservoir for resistant bacteria and a source of environmental contamination. Antimicrobial resistance patterns of ESBLproducing $E$. coli detected in all sample types and study locations were quite similar. In almost all ESBL-producing $E$. coli isolates, resistance was shown against ampicillin, erythromycin, ceftriaxone, tetracycline and chloramphenicol. Moreover, multidrug resistance was found in all isolates of ESBL-producing E. coli. The differences in antimicrobial agent resistance patterns can be used to differentiate sources by employing analytical tools such as discriminant function analysis. A molecular typing protocol is recommended for use in a discriminant function analysis for pattern determination of pathogen spreading. However, genetic fingerprinting techniques for microbial source tracking are more expensive, and facilities with appropriate equipment and expertise are required.
\end{abstract}

Keywords: ESBL-producing E. coli, prevalence, antimicrobial resistance, pig farm, layer farm. 
A. Nuangmek, S. Rojanasthien, S. Chotinun, et al. 2018. Antimicrobial Resistance in ESBL-Producing Escherichia coli Isolated

\section{INTRODUCTION}

Antimicrobial resistance has been recognized as an emerging global problem in both animals and humans, with enormous health and economic impacts [13,25]. This phenomenon has been caused mainly by the growing prevalence of extended-spectrum beta-lactamase (ESBL)-producing Enterobacteriaceae [5,9,10] and has resulted in increased use of last-resort antimicrobial drugs, e.g. carbapenems and colistin [18]. ESBL-producing Escherichia coli have been isolated with increasing frequency from human and animal samples, a development that has drawn considerable attention worldwide [6,24]. Food-producing animals and their surrounding environment colonized with ESBL-producing $E$. coli have been considered to be potential sources of resistant bacterial infections in the community [24].

In Thailand, the pig and poultry industries have become the largest food-producing animal sectors. Pig production has the highest total consumption of antimicrobials (66.96\%), followed by layer production (3.47\%) [1]. Chiang Mai-Lamphun (northern Thailand) and Chon Buri provinces (eastern Thailand) were chosen as the focus of this study because they have a high density of animal raising, with $46.57 \%$ of the layers and $26.57 \%$ of the pigs in the country [11].

The objective of this study was to determine the prevalence and identify patterns of antimicrobialresistant ESBL-producing E. coli isolated from pigs, layer chickens, farm workers and the environment (stagnant water), in order to increase awareness about antimicrobial usage and to understand the growth dynamics of antimicrobial resistance on farms.

\section{MATERIALS AND METHODS}

\section{Study design and sample size determination}

A cross-sectional study was conducted in layer and pig farms in Chiang Mai-Lamphun and Chon Buri provinces during May 2015 to April 2016. The sample size of this study was determined using Epi Info ${ }^{\mathrm{TM}} 7$ software. Expected prevalence of $10 \%$, with $95 \%$ confidence interval and $5 \%$ error, were used as the parameters for calculation [12]. Considered for the population on pig and layer farms, 515 and 225 are included, respectively. The sample sizes for pig and layer farms were 107 and 89. Fifty-nine pig farms and 54 layer farms in Chiang Mai-Lamphun and 48 pig farms and 35 layer farms in Chon Buri province were selected using a convenience sampling method. The data of antimicrobial use on farms were collected by questionnaire.

Sample collection

The samples in this study consisted of animal feces, stools of farm workers, and water from the environment. Fecal samples from pigs and layers were collected rectally from healthy animals using culture swabs $\left(\text { Culturette }{ }^{\circledR}\right)^{1}$. Five rectal swab samples from the same pig group were pooled into one sample, the same as with cloacal swab samples from layers. Stool samples were collected from healthy farm workers who had been working at the site for more than one year. Cary-Blair transport media (non-nutritive medium) tubes were used to store all swab specimens. Farm environment samples (stagnant water, $30 \mathrm{~mL}$ each) were collected from both pig and layer farms. All samples were kept in an icebox before transporting to the Faculty of Medicine Siriraj Hospital laboratory.

\section{ESBL-producing E. coli cultures and antimicrobial sus-} ceptibility testing

Stool samples from healthy adult farm workers, rectal or cloacal swabs from healthy animals, and stagnant water samples from pig and layer farms were cultured on MacConkey agar and incubated at $35^{\circ} \mathrm{C}$ for $24 \mathrm{~h}$. Suspected colonies on MacConkey agar were red in color with a surrounding dark red area of precipitated bile salts; presumptive colonies were confirmed by a biochemical test. ESBL-producing $E$. coli isolates were detected using a double-disk diffusion method according to EUCAST (European Committee on Antimicrobial Susceptibility Testing) guidelines.

An extra zone was determined between a disk of amoxicillin/clavulanate $(20 \mathrm{mg} / 10 \mathrm{mg})$ and a $30 \mathrm{mg}$ disk of each cephalosporin (ceftriaxone, ceftazidime and cefepime) placed at a distance of $20 \mathrm{~mm}$ from center to center of the disks on Mueller-Hinton agar plates. Clear extension of the edge of the cephalosporin inhibition zone toward the amoxicillin/clavulanate disk was interpreted as positive for ESBL production [12]. All of the ESBL-producing E. coli isolates were tested for antimicrobial susceptibility, using a disk diffusion method (BD BBL ${ }^{\mathrm{TM}}$ Sensi-Disc ${ }^{\mathrm{TM}}$ antimicrobial susceptibility test discs) ${ }^{1}$. Tests were conducted on ceftriaxone (CRO) $30 \mu \mathrm{g}$, cefoxitin (FOX) $30 \mu \mathrm{g}$, ceftazidime (CAZ) 30 $\mu \mathrm{g}$, imipenem (IPM) $10 \mu \mathrm{g}$, meropenem (MEM) $10 \mu \mathrm{g}$, gentamicin $(\mathrm{CN}) 10 \mu \mathrm{g}$, amikacin (AK) $30 \mu \mathrm{g}$, nalidixic acid (NA) $30 \mu \mathrm{g}$, trimethoprim/sulfamethoxazole (SXT) 
$1.25 / 23.75 \mu \mathrm{g}$, tetracycline (TE) $30 \mu \mathrm{g}$, erythromycin (E) $15 \mu \mathrm{g}$, ampicillin (AMP) $10 \mu \mathrm{g}$, amoxicillin/clavulanate (AMC) 20/10 $\mu$ g, chloramphenicol (C) $30 \mu \mathrm{g}$ and colistin (CT) $10 \mu \mathrm{g}$. Antimicrobial susceptibility testing was performed according to the guidelines of the Clinical Laboratory Standards Institute (CLSI) [8].

\section{Data analysis}

The data were described by descriptive statistics and associations between the sample collection sites (Chiang Mai-Lamphun provinces vs Chon Buri province). Antimicrobial drug resistance was expressed as odds ratios with 95\% confidence interval using Epi Info ${ }^{\mathrm{TM}} 7$ software [7].

\section{RESULTS}

A total of 588 samples were collected, from which 216 (36.7\%) ESBL-producing E. coli isolates were retrieved. Most were pig-associated samples $(51.1 \% ; 164 / 321)$; of these, approximately $75 \%$ (80/107) originated from healthy pigs, followed by $57.0 \%$ (61/107) and 21.5\% (23/107) from pig farm workers and stagnant water, respectively. In layer-associated samples, $19.4 \%$ (52/267) were positive; $51.7 \%$ (46/89) were from farm worker stool samples and $6.7 \%(6 / 89)$ were detected in healthy layer rectal swab samples. None of the stagnant water samples from layer farms were found to contain ESBL-producing E. coli isolates (Table 1).

Most of the ESBL-producing E. coli isolates were resistant to ampicillin (99.5\%), followed by erythromycin $(98.6 \%)$, ceftriaxone $(96.3 \%)$, tetracycline $(85.6 \%)$ and chloramphenicol $(75.5 \%)$. Colistin-resistant isolates were present only in layer farm workers $(2.2 \%)$, while imipenem and meropenem were $100 \%$ effective against ESBL-producing E. coli isolates from all types of samples (Table 2).

ESBL-producing $E$. coli isolates resistant to $>3$ antimicrobial agents were denoted as multidrugresistant strains. Distribution of the ten most common antimicrobial resistance patterns of multidrug-resistant ESBL-producing E. coli isolates from animals (pigs and layers), farm workers and the environment (stagnant water) is shown in Fig. 1. Drug resistance patterns of major ESBL-producing E. coli phenotypes were AMP-CROE-TE-C-SXT-CN (14.4\%), followed by AMP-CRO-ETE-C-SXT-NA-CN (11.6\%), AMP-CRO-E-TE-C-CN $(5.6 \%)$ and AMP-CRO-E-TE-C-SXT-CN-CAZ (5.6\%), respectively. The strains derived from animals, humans and the environment were grouped in identical drug resistance pattern phenotypes.

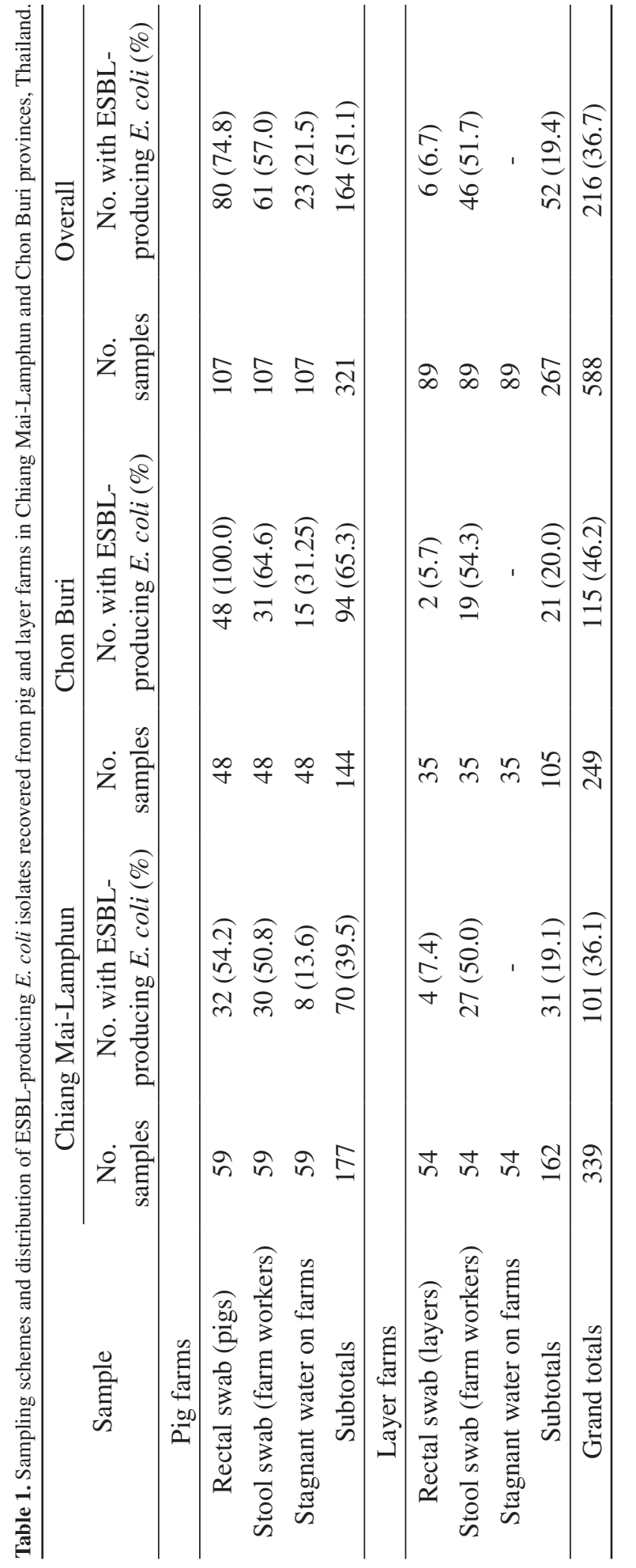

Tiamulin was the most common antimicrobial used on pig farms in Chiang Mai-Lamphun (83.1\%), followed by amoxicillin (71.2\%), tylosin $(50.8 \%)$ and chlortetracycline $(23.7 \%)$, while the antimicrobials most commonly used on pig farms in Chon Buri were 
amoxicillin (54.2\%), tiamulin (45.8\%), colistin (25.0\%) and penicillin/streptomycin (25.0\%). The most widely used antimicrobials on layer farms in Chiang MaiLamphun were tiamulin $(75.0 \%)$, chlortetracycline $(64.3 \%)$, tylosin $(55.4 \%)$ and amoxicillin $(25.0 \%)$, while the antimicrobials most commonly used in Chon Buri were tylosin (91.4\%), amoxicillin (62.9\%), tiamulin $(28.6 \%)$ and colistin $(28.6 \%)$ [Table 3].
Generally, macrolides were the most common agents used over our study areas.

Odds ratios between sample collection sites (Chiang Mai-Lamphun vs Chon Buri) and resistance results (resistant vs susceptible) against each antimicrobial agent most commonly reported in the study were analyzed. No significant differences were detected for all sample types (Table 4).

Table 2. Percent resistance against each antimicrobial agent of ESBL-producing E. coli isolates originating from different sources in pig and layer farms in Chiang Mai-Lamphun and Chon Buri provinces.

\begin{tabular}{|c|c|c|c|c|c|c|}
\hline \multirow{3}{*}{ Antimicrobial agent } & \multicolumn{6}{|c|}{ Percent resistance against each antimicrobial agent } \\
\hline & \multicolumn{3}{|c|}{ Pig farms } & \multicolumn{2}{|c|}{ Layer farms } & \multirow{2}{*}{$\begin{array}{c}\text { Overall } \\
(\mathrm{n}=216)\end{array}$} \\
\hline & $\begin{array}{c}\text { Pigs } \\
(\mathrm{n}=80)\end{array}$ & $\begin{array}{l}\text { Farm workers } \\
\quad(n=61)\end{array}$ & $\begin{array}{l}\text { Stagnant water } \\
(\mathrm{n}=23)\end{array}$ & $\begin{array}{l}\text { Layers } \\
(n=6)\end{array}$ & $\begin{array}{l}\text { Farm workers } \\
\quad(n=46)\end{array}$ & \\
\hline Ampicillin & 100.0 & 100.0 & 100.0 & 100.0 & 97.8 & 99.5 \\
\hline Erythromycin & 100.0 & 98.4 & 95.7 & 100.0 & 97.8 & 98.6 \\
\hline Ceftriaxone & 96.3 & 93.4 & 95.7 & 100.0 & 100.0 & 96.3 \\
\hline Tetracycline & 83.6 & 80.3 & 95.7 & 66.7 & 93.5 & 85.6 \\
\hline Chloramphenicol & 81.3 & 68.9 & 82.6 & 100.0 & 67.4 & 75.5 \\
\hline Gentamicin & 77.5 & 54.1 & 95.7 & 100.0 & 60.8 & 69.9 \\
\hline Trimethoprim/Sulfamethoxazole & 66.3 & 54.1 & 69.6 & 33.3 & 60.8 & 61.1 \\
\hline Nalidixic acid & 45.0 & 49.2 & 43.5 & 50.0 & 58.7 & 49.1 \\
\hline Ceftazidime & 20.0 & 16.4 & 13.0 & 16.7 & 32.6 & 20.8 \\
\hline Amoxicillin/Clavulanic acid & 6.3 & 4.9 & - & 16.7 & 21.7 & 8.8 \\
\hline Cefoxitin & 5.0 & 4.9 & 4.3 & 16.7 & 2.2 & 4.6 \\
\hline Amikacin & - & - & 4.3 & - & 2.2 & 0.9 \\
\hline Colistin & - & - & - & - & 2.2 & 0.5 \\
\hline Imipenem & - & - & - & - & - & - \\
\hline Meropenem & - & - & - & - & - & - \\
\hline
\end{tabular}

Table 3. Antimicrobial agents used in routine practices on pig and layer farms in Chiang Mai-Lamphun and Chon Buri provinces during the preceding year.

\begin{tabular}{|c|c|c|c|c|}
\hline \multirow{3}{*}{ Antimicrobial agent } & \multicolumn{4}{|c|}{ No. of farms using each antimicrobial agent (\%) } \\
\hline & \multicolumn{2}{|c|}{ Pig farms } & \multicolumn{2}{|c|}{ Layer farms } \\
\hline & $\begin{array}{l}\text { Chiang Mai-Lamphun } \\
(\mathrm{n}=59)\end{array}$ & $\begin{array}{l}\text { Chon Buri } \\
(\mathrm{n}=48)\end{array}$ & $\begin{array}{l}\text { Chiang Mai-Lamphun } \\
(\mathrm{n}=54)\end{array}$ & $\begin{array}{c}\text { Chon Buri } \\
(\mathrm{n}=35)\end{array}$ \\
\hline Tiamulin & $49(83.1)$ & $22(45.8)$ & $42(75.0)$ & $10(28.6)$ \\
\hline Amoxicillin & $42(71.2)$ & $26(54.2)$ & $14(25.0)$ & $22(62.9)$ \\
\hline Penicillin/Streptomycin & $39(66.1)$ & $12(25.0)$ & - & - \\
\hline Tylosin & $30(50.8)$ & $5(11.4)$ & $31(55.4)$ & $32(91.4)$ \\
\hline Oxytetracycline & $28(47.5)$ & $4(8.3)$ & - & - \\
\hline Lincomycin & $21(35.6)$ & $1(2.1)$ & - & - \\
\hline Colistin & $18(30.5)$ & $12(25.0)$ & $4(7.1)$ & $10(28.6)$ \\
\hline Trimethoprim/Sulfamethoxazole & $16(27.1)$ & $1(2.1)$ & $1(1.8)$ & - \\
\hline Chlortetracycline & $14(23.7)$ & $7(14.6)$ & $36(64.3)$ & $6(17.1)$ \\
\hline Cefotaxime & $9(15.3)$ & $7(14.6)$ & - & - \\
\hline Enrofloxacin & 7 (11.9) & $4(8.3)$ & $7(12.5)$ & $3(8.6)$ \\
\hline Kanamycin & $3(5.1)$ & - & - & - \\
\hline Gentamicin & $12(0.3)$ & 7 (14.6) & $2(3.6)$ & $3(8.6)$ \\
\hline Doxycycline & - & $1(2.1)$ & $1(1.8)$ & $3(8.6)$ \\
\hline
\end{tabular}


A. Nuangmek, S. Rojanasthien, S. Chotinun, et al. 2018. Antimicrobial Resistance in ESBL-Producing Escherichia coli Isolated from Layer and Pig Farms in Thailand.

Acta Scientiae Veterinariae. 46: 1538.

Table 4. Odds ratios between sample collection sites and resistance results against each antimicrobial agent most commonly reported in the study.

\begin{tabular}{|c|c|c|c|c|c|c|}
\hline \multirow{2}{*}{ Sample } & \multicolumn{6}{|c|}{ Odds ratio ( $95 \%$ confidence interval)* } \\
\hline & Ampicillin & Erythromycin & Ceftriaxone & Tetracycline & Chloramphenicol & Gentamicin \\
\hline \multicolumn{7}{|l|}{ Pig farms } \\
\hline Rectal swab (pigs) & NA & NA & NA & $\begin{array}{c}2.39 \\
(0.60-9.50)\end{array}$ & $\begin{array}{c}0.34 \\
(0.11-1.08)\end{array}$ & $\begin{array}{c}0.73 \\
(0.26-2.13)\end{array}$ \\
\hline $\begin{array}{c}\text { Stool swab } \\
\text { (farm workers) }\end{array}$ & NA & NA & $\begin{array}{c}0.96 \\
(0.13-7.33)\end{array}$ & $\begin{array}{c}0.63 \\
(0.18-2.27)\end{array}$ & $\begin{array}{c}1.24 \\
(0.40-3.83)\end{array}$ & $\begin{array}{c}1.60 \\
(0.58-4.41)\end{array}$ \\
\hline $\begin{array}{c}\text { Stagnant water on } \\
\text { farms }\end{array}$ & NA & NA & NA & NA & $\begin{array}{c}1.75 \\
(0.15-20.23) \\
\end{array}$ & NA \\
\hline \multicolumn{7}{|l|}{ Layer farms } \\
\hline Rectal swab (layers) & NA & NA & NA & NA & NA & NA \\
\hline $\begin{array}{c}\text { Stool swab } \\
\text { (farm workers) }\end{array}$ & NA & NA & NA & $\begin{array}{c}3.06 \\
(0.26-36.42)\end{array}$ & NA & $\begin{array}{c}0.85 \\
(0.25-2.84)\end{array}$ \\
\hline
\end{tabular}

$\mathrm{NA}=$ not available. $*$ Chon Buri was used as the reference category for the sample collection sites.

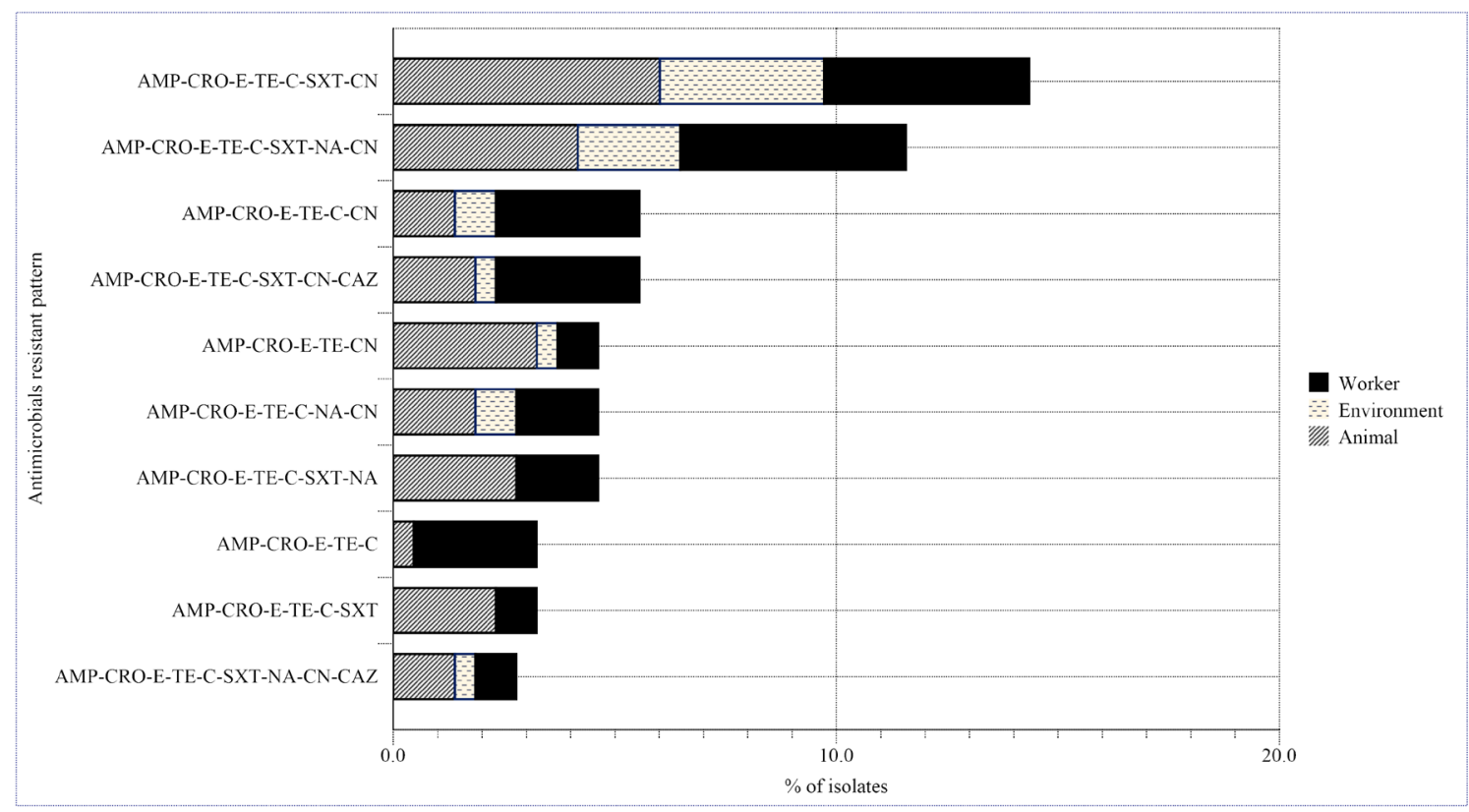

Figure 1. Distribution of ten most common antimicrobial resistance patterns of multidrug-resistant ESBL-producing E. coli isolates separated in each source. *amikacin (AK); ampicillin (AMP); amoxicillin/clavulanate (AMC); ceftazidime (CAZ); cefoxitin (FOX); ceftriaxone (CRO); chloramphenicol (C); colistin (CT); erythromycin (E); gentamicin (CN); imipenem (IPM); meropenem (MEM); nalidixic acid (NA); tetracycline (TE) and trimethoprim/sulfamethoxazole (SXT).

\section{DISCUSSION}

The prevalence of ESBL-producing E. coli in fecal samples from healthy pigs $(74.8 \%)$ was higher compared with healthy layers $(6.7 \%)$, which was similar to the prevalence found in a previous study $[1,3]$. The large amount and variety of antimicrobials used on pig farms could indicate that pig farms are a common major source of resistant ESBL-producing $E$. coli [4]. This is at variance with the results of studies by Hiroi et al. and Geser et al., in which layers are recognized as the major reservoir of ESBL-producing E. coli $[14,15]$.

Upon the comparison of each sample type, the prevalence of ESBL-producing E. coli in samples from a farm environment was lower than in samples of animal origin. Indicating the livestock function is 
known to be a reservoir for resistant bacteria and a source of environmental contamination [22]. However, no stagnant water samples on layer farms were identified as containing ESBL-producing E. coli. Less water is utilized for a layer operation compared with the requirements for pigs. Water is also drained rapidly, so pathogen accumulation in stagnant water is not likely to occur in layer farms. Interestingly, in layerassociated samples, the prevalence of ESBL-producing E. coli in farm worker samples was found to be at a higher level compared with samples from healthy layers. Contamination may possibly be associated with non-farm sources.

When considering geographic areas, the ESBL-producing E. coli prevalence observed in the eastern region of the country was higher than in the north, in contrast with a previous report [3]. The higher prevalence in the north could be a result of variations in study design, different local environments and/or animal production practices $[3,19,23]$.

Antimicrobial resistance profiles of ESBLproducing $E$. coli collected from all sample types were similar (Table 2). Most of the isolates were resistant against ampicillin, erythromycin, ceftriaxone, tetracycline and chloramphenicol. Moreover, ampicillin-, erythromycin- and ceftriaxone-resistant isolates were detected almost equally in animals, humans and the environment. These antimicrobials have been widely applied in animal production in Thailand. Improper use could have created selective pressure, resulting in a high antimicrobial resistance rate [16].

Tetracycline and chloramphenicol resistance in ESBL-producing E. coli was found at a low level. In Thailand, tetracycline usage in food-producing animals has been restricted since 2003 [21], while chloramphenicol usage has been prohibited since 2002 [20]. Consequently, selective pressure is unlikely. Horizontal gene transfer, facilitating the spread of resistant genes within and between various bacterial species, is possible [17]. Remarkably, ESBL-producing $E$. coli resistant to colistin were present in layer farm workers $(2.2 \%)$. Colistin is a last-resort antimicrobial for treatment of severe infections caused by multidrug-resistant gram-negative bacteria, in particular those caused by carbapenemresistant Klebsiella pneumoniae, Acinetobacter baumannii and Pseudomonas aeruginosa [2]. This situation makes it imperative to evaluate the magnitude of resistance to colistin in both humans and animals and to implement a system for surveillance of colistin resistance.

All ESBL-producing E. coli detected in the study were multidrug-resistant. AMP-CRO-E-TE-CSXT-CN and AMP-CRO-E-TE-C-SXT-NA-CN were the major phenotypic patterns, including strains derived from animals, humans and the environment. The transmission of pathogens among originating sources (animal/human/environment) is a possibility. A molecular typing protocol is strongly recommended for use in a discriminant function analysis for pattern determination of pathogen spreading [22].

\section{CONCLUSION}

The prevalence of ESBL-producing E. coli on pig farms was higher than on layer farms; contamination rates in Chon Buri province tended to be higher. Antimicrobial resistance patterns of ESBLproducing $E$. coli detected in all types and sites of samples were comparable. Multidrug resistance was found in all isolates of ESBL-producing E. coli. Isolates originating from animals, farm workers and the environment were demonstrated to have identical drug resistance patterns. Awareness regarding antimicrobial usage on farms should be addressed. Furthermore, comprehensive surveillance systems to monitor antimicrobial resistance ought to be established in order to reduce the selective pressure downstream on humans.

\section{MANUFACTURER}

${ }^{1}$ Becton, Dickinson and Company. Sparks, MD, USA.

Funding. International Development Research Centre (Canada): project number 106915-005.

Acknowledgements. The authors are grateful to the participants and staff members of Chiang Mai-Lamphun and Chon Buri Provincial Livestock Offices for their help in sample and data collection.

Ethical approval. The experimental procedures were approved by the Faculty of Veterinary Medicine, Chiang Mai University (approval number: R10/2556).

Declaration of interest. The authors report no conflicts of interest. The authors alone are responsible for the content and writing of the paper. 


\section{REFERENCES}

1 Animal Health Products Association. 2013. AHPA Market information. Samut Sakhon: A.T. Printing Co., 451p.

2 Biswas S., Brunel J.M., Dubus J.C., Reynaud-Gaubert M. \& Rolain J.M. 2012. Colistin: an update on the antibiotic of the 21st century. Expert Review of Anti-Infective Therapy. 10(8): 917-934.

3 Boonyasiri A., Tangkoskul T., Seenama C., Saiyarin J., Tiengrim S. \& Thamlikitkul V. 2014. Prevalence of antibiotic resistant bacteria in healthy adults, foods, food animals, and the environment in selected areas in Thailand. Pathogens and Global Health. 108(5): 235-245.

4 Cameron A. \& McAllister T.A. 2016. Antimicrobial usage and resistance in beef production. Journal of Animal Science and Biotechnology. 7: 68.

5 Cantón R., Novais A., Valverde A., Machado E., Peixe L., Baquero F. \& Coque T.M. 2008. Prevalence and spread of extended-spectrum beta-lactamase-producing Enterobacteriaceae in Europe. Clinical Microbiology and Infection. 14(1): 144-153.

6 Carattoli A. 2008. Animal reservoirs for extended spectrum beta-lactamase producers. Clinical Microbiology and Infection. 14(1): 117-123.

7 Centers for Disease Control and Prevention. Epi Info ${ }^{\text {TM }}$. 2014. Available at: $<$ http://wwwn.cdc.gov/epiinfo/7/index. htm.>. [Accessed online in December 2014].

8 Clinical and Laboratory Standards Institute. 2013. Performance standards for antimicrobial susceptibility testing; twenty-first informational supplement (CLSI document M100-S21). Wayne: CLSI, 163p.

9 Coque T.M., Baquero F. \& Canton R. 2008. Increasing prevalence of ESBL-producing Enterobacteriaceae in Europe. Eurosurveillance. 13(47): 19044.

10 Dahms C., Hübner N.O., Kossow A., Mellmann A., Dittmann K. \& Kramer A. 2015. Occurrence of ESBL-producing Escherichia coli in livestock and farm workers in Mecklenburg-Western Pomerania, Germany. PLoS One. 10(11).

11 Department of Livestock Development, Thailand. 2013. Thai livestock farmer database system. Available at: <http:// survey-c.dld.go.th>. [Accessed online in December 2016].

12 European Committee on Antimicrobial Susceptibility Testing. 2013. EUCAST guidelines for detection of resistance mechanisms and specific resistances of clinical and/or epidemiological importance. pp.11-19. Available at: <http:// www.amcli.it/wpcontent/uploads/2015/10/EUCAST_detection_resistance_mechanisms_V1.pdf>. [Accessed online in September 2014].

13 Gandra S., Barter D.M. \& Laxminarayan R. 2014. Economic burden of antibiotic resistance: how much do we really know? Clinical Microbiology and Infection. 20(10): 973-980.

14 Geser N., Stephan R. \& Hächler H. 2012. Occurrence and characteristics of extended-spectrum $\beta$-lactamase (ESBL) producing Enterobacteriaceae in food producing animals, minced meat and raw milk. BMC Veterinary Research. 8: 21.

15 Hiroi M., Yamazaki F., Harada T., Takahashi N., Iida N., Noda Y., Yagi M., Nishio T., Kanda T., Kawamori F., Sugiyama K., Masuda T., Hara-Kudo Y. \& Ohashi N. 2012. Prevalence of extended-spectrum $\beta$-lactamase-producing Escherichia coli and Klebsiella pneumoniae in food-producing animals. Journal of Veterinary Medical Science. 74(2): 189-195.

16 Horton R.A., Randall L.P., Snary E.L., Cockrem H., Lotz S., Wearing H., Duncan D., Rabie A., McLaren I., Watson E., La Ragione R.M. \& Coldham N.G. 2011. Fecal carriage and shedding density of CTX-M extended-spectrum B-lactamase-producing Escherichia coli in cattle, chickens, and pigs: implications for environmental contamination and food production. Applied and Environmental Microbiology. 77(11): 3715-3719.

17 Kelly B.G, Vespermann A. \& Bolton D.J. 2009. The role of horizontal gene transfer in the evolution of selected foodborne bacterial pathogens. Food and Chemical Toxicology. 47(5): 951-968.

18 Liu Y.Y., Wang Y., Walsh T.R., Yi L.X., Zhang R., Spencer J., Doi Y., Tian G., Dong B., Huang X., Yu L.F., Gu D., Ren H., Chen X., Lv L., He D., Zhou H., Liang Z., Liu J.H. \& Shen J. 2016. Emergence of plasmid-mediated colistin resistance mechanism MCR-1 in animals and human beings in China: a microbiological and molecular biological study. Lancet Infectious Diseases. 16(2): 161-168.

19 Luvsansharav U.O., Hirai I., Niki M., Sasaki T., Makimoto K., Komalamisra C., Maipanich W., Kusolsuk T., Sa-Nguankiat S., Pubampen S. \& Yamamoto Y. 2011. Analysis of risk factors for a high prevalence of extendedspectrum $\beta$-lactamase-producing Enterobacteriaceae in asymptomatic individuals in rural Thailand. Journal of Medical Microbiology. 60: 619-624. 
20 Ministry of Agriculture and Cooperatives, Thailand. 2002. Announcement of the Ministry of Agriculture and Cooperatives. B.E. 2545. Dated 4th June B.E. 2545 (2002), 2p. Available at: < http://www.dld.go.th/th/images/stories/ law/announce_animalfeed2545.pdf>. [Accessed online in March 2017].

21 Ministry of Agriculture and Cooperatives, Thailand. 2003. Announcement of the Ministry of Agriculture and Cooperatives. B.E. 2546. Dated 4th June B.E. 2546 (2003). Available at:: <http://www.dld.go.th/th/index.php/th/legaldld-menu/law06-menu/133-8-2546>. [Accessed online in December 2016].

22 Sasaki T., Hirai I., Niki M., Nakamura T., Komalamisra C., Maipanich W., Kusolsuk T., Sa-Nguankiat S., Pubampen S. \& Yamamoto Y. 2010. High prevalence of CTX-M beta-lactamase-producing Enterobacteriaceae in stool specimens obtained from healthy individuals in Thailand. Journal of Antimicrobial Chemotherapy. 65(4): 666-668.

23 Sayah R.S., Kaneene J.B., Johnson Y., Miller R. 2005. Patterns of antimicrobial resistance observed in Escherichia coli isolates obtained from domestic- and wild-animal fecal samples, human septage, and surface water. Applied and Environmental Microbiology. 71(3): 1394-1404.

24 Smet A., Martel A., Persoons D., Dewulf J., Heyndrickx M., Herman L., Haesebrouck F. \& Butaye P. 2010. Broad-spectrum $\beta$-lactamases among Enterobacteriaceae of animal origin: molecular aspects, mobility and impact on public health. FEMS Microbiology Reviews. 34(3): 295-316.

25 World Health Organization. 2001. Global Strategy for Containment of Antimicrobial Resistance. Geneva: WHO, 99p. 